\title{
Analytical Solutions for the Breakdown Voltages of Punched-Through Diodes Having Curved Junction Boundaries at the Edges
}

\author{
V. ANANTHARAM AND K. N. BHAT
}

\begin{abstract}
Analytical expressions are derived for the breakdown voltages of punched-through diodes having a plane structure terminated with cylindrical and spherical curved boundaries at the edges, through the use of suitable approximations for the electric field in the depletion layer. The expressions derived include both $\mathrm{p}^{+}-\mathrm{i}-\mathrm{n}^{+}$and $\mathrm{p}^{+}-\mathrm{p}-\mathrm{n}^{+}$(or $\left.\mathrm{p}^{+}-\mathrm{n}^{+} \mathrm{n}^{+}\right)$types and are given in terms of the middle-region (i-layer or p-layer) width, the radius of curvature of the junction edge, the punchthrough voltage, and the plane parallel breakdown voltage of $\mathrm{p}^{+} \cdot \mathrm{i}-\mathrm{n}^{+}$ diodes.

The results obtained include a correlation between the middle-region (p-layer) width and the width of the depletion layer in the curved portions of the junction when the applied reverse bias across the diode is just sufficient so that punchthrough takes in the portions where the junction is plane parallel. These results are made use of in the breakdown voltage calculations.
\end{abstract}

\section{INTRODUCTION}

$I^{2}$ T IS VERY WELL KNOWN that the junctions fabricated by diffusing impurities through windows in an impervious masking layer, to form a "planar" junction in the substrate material, result in a plane structure terminated with cylindrical curved boundaries at the window edge, and that this curvature takes spherical shape if the window has sharp corners. Theoretical design curves relating the breakdown voltage to the radius of curvature and substrate doping, experimental verification of the results, and the extension of the results to diffused junctions have been discussed in detail in the literature [1]-[9] for the case of p-n junction diodes. Recently [10], analytical solutions for the breakdown voltages of abrupt cylindrical and spherical junctions using simplified expressions for the ionization integral [11] have been published. How. ever, in literature, no attempt has been made to obtain closedform solutions or computer solutions to give design curves which ease the calculation of the breakdown voltage of "punched-through diodes" with cylindrical and spherical boundaries.

The purpose of the present paper is to extend the concepts [10] of curvature-limited breakdown voltages of cylindrical and spherical $p$-n junctions to obtain analytical solutions for the breakdown voltage of "punched-through diodes" namely $\mathrm{p}^{+}-\mathrm{i}-\mathrm{n}^{+}$diodes and $\mathrm{p}^{+}-\mathrm{p}-\mathrm{n}^{+}$(or $\mathrm{p}^{+}-\mathrm{n}-\mathrm{n}^{+}$) diodes with abrupt junctions. The structure of the diode considered for the present analysis is shown in Fig. 1(a). The depletion layer in this

Manuscript received May 7, 1979; revised December 6, 1979.

V. Anantharam is with the Department of Electrical Engineering, Indian Institute of Technology, Madras 600036, India.

K. N. Bhat is with the Electrical and Systems Engineering Department, Rensselaer Polytechnic Institute, Troy, NY 12181, on leave from the Indian Institute of Technology, Madras, India.

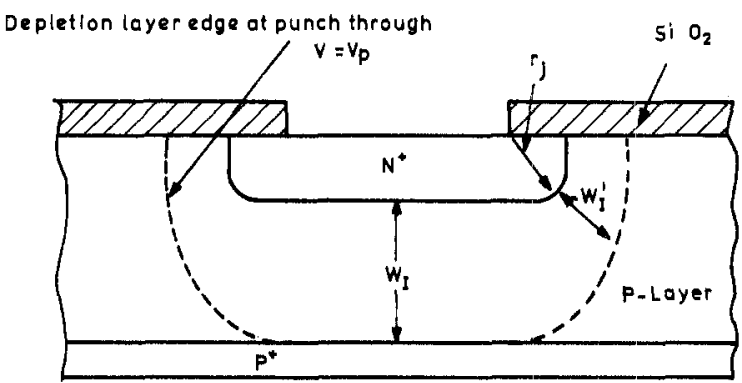

(a)

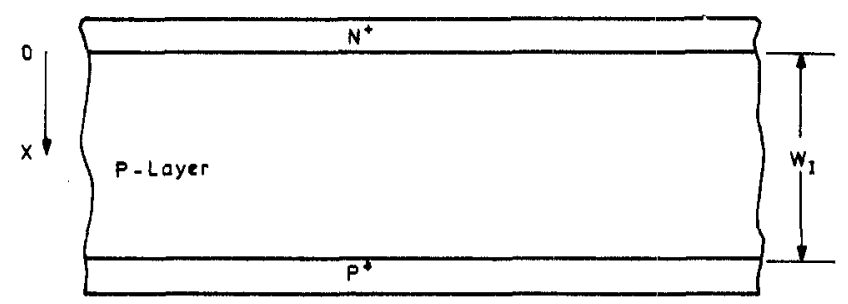

(b)

Fig. 1. (a) The structure of the punched-through diode. (b) Punchedthrough diode with plane-parallel junctions.

structure punches through the middle layer ( $\mathrm{player}$ or $\mathrm{n}$ layer) at voltages below the breakdown voltage. The breakdown voltage is described by the avalanche multiplication at the curved boundaries. As done earlier [10], a simple one-dimensional approach of integration along a radius vector is carried out for determining the voltages across the depletion layer in the curved portions and by equating this to the punchthrough voltage $V_{P}$ of the plane portion of the diode, the width $W_{I}^{\prime}$ of the depletion layer at the curved portions [see Fig. 1(a)] for a voltage equal to $V_{P}$, is related to the width $W_{I}$ of the middle region in the plane parallel portion of the diode. If $r_{j}$ is the radius of curvature of the junction at the edge, it is shown that $W_{I}^{\prime} / r_{j}$ is decided by the $W_{I} / r_{j}$ ratio and the $W_{I}^{\prime} / r_{j}$ ratio is calculated as a function of $W_{I} / r_{j}$ for cylindrical and spherical curvatures separately. The analytical solutions for the breakdown voltages are given in terms of $W_{I}^{\prime} / r_{j}$ ratio, the planeparallel punchthrough voltage $V_{P}$, and the breakdown voltage $B V_{P P I}$ of the $\mathrm{p}^{+} \cdot \mathrm{i}-\mathrm{n}^{+}$structure having plane-parallel junctions and i-layer width of $W_{I}$.

The results hold good for $\mathrm{p}^{+}-\mathrm{p} \cdot \mathrm{n}^{+}\left(\right.$or $\left.\mathrm{p}^{+}-\mathrm{n}-\mathrm{n}^{+}\right)$diodes, which punchthrough before breakdown, for any radius of curvature, p-layer width, and for the doping concentrations of practical interest. The results are plotted and can be used very conveniently for the breakdown-voltage calculations in the design of punched-through diodes. 


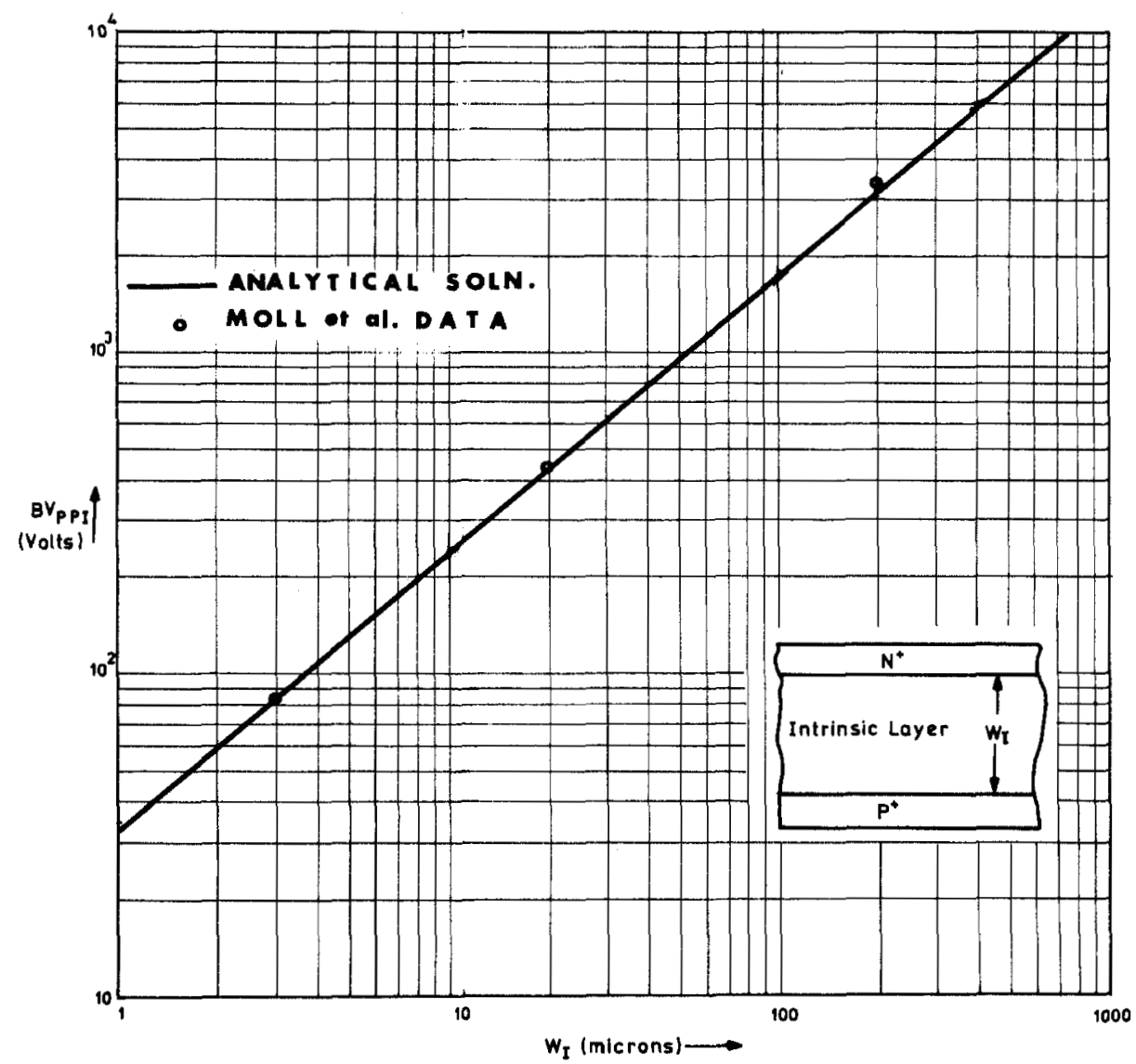

Fig. 2. Breakdown voltage of abrupt plane-parallel junction $\mathrm{p}^{+}-\mathrm{i}-\mathrm{n}^{+}$diodes.

\section{Parallel-Plane Punched-Through Junction}

This section considers the breakdown voltage of $\mathrm{p}^{+}-\mathrm{i}-\mathrm{n}^{+}$ diodes with plane-parallel abrupt junctions. Referring to Fig. 1(b), when the applied voltage is equal to the punchthrough voltage $V_{P}$, the electric field $E_{1}(x)$ is given by

$$
E_{1}(x)=\frac{2 V_{P}}{W_{I}}\left(1-\frac{x}{W_{I}}\right)
$$

where

$$
V_{P}=\frac{q N_{I} W_{I}^{2}}{2 \epsilon_{r} \epsilon_{0}}
$$

$N_{I}$ is the doping concentration in the middle layer, $\epsilon_{r}$ is the relative permittivity, $\epsilon_{0}$ is the free-space dielectric constant, and $q$ is the electronic charge.

When the applied voltage $V$ is greater than $V_{P}$, the additional voltage $\left(V-V_{P}\right)$ drops uniformly over the $i$ layer and hence the electric field $E(x)$ takes the form

$$
E(x)=\frac{2 V_{P}}{W_{I}}\left(1-\frac{x}{W_{I}}\right)+\frac{V-V_{P}}{W_{I}} .
$$

Using the concepts of average ionization coefficient, the breakdown voltage is obtained by solving the ionization integral

$$
\int_{0}^{W_{I}} \alpha(x) d x=1
$$

where $\alpha(x)$ depends on $E(x)$, as given by Fulop [11]

$$
\alpha=A E^{7} \mathrm{~cm}^{-1}
$$

where

$$
A=1.8 \times 10^{-35} \text {. }
$$

Evaluating the integral for a $\mathrm{p}^{+}-\mathrm{i}-\mathrm{n}^{+}$diode, whose middle layer is purely intrinsic and $V_{P}=0$, the plane-parallel breakdown voltage $B V_{P P I}$ (the suffix $I$ denoting intrinsic) is given by

$$
B V_{P P I}=\left(\frac{W_{I}^{6}}{A}\right)^{1 / 7} \text {. }
$$

This expression for the breakdown voltage also holds good for $\mathrm{p}^{+}$-p-n ${ }^{+}$diodes having $V_{P} \ll B V_{P P}$. Fig. 2 gives a plot of $B V_{P P I}$ as a function of the i-layer width $W_{I}$. This result is in very good agreement with the values given by Moll et al. [12] for $\mathrm{p}^{+}-\mathrm{i}-\mathrm{n}^{+}$diodes. Moll's results are marked in Fig. 2 for comparison. From this it is clear that Fulop's formula for ionization coefficient can be successfully used to calculate breakdown voltage of punched-through diodes as well. Therefore, this form has been used in subsequent sections and $B V_{P P I}$ is used as the normalizing parameter.

\section{Punched-Through Diode with Plane Junction Terminated with Cylindrical Boundary}

Considering the structure with abrupt junctions, shown in Fig. 1(a), when the applied voltage is $V_{P}$, the depletion layer reaches the $p^{+}-p$ boundary in the plane-parallel portions and the width of the depletion layer in the cylindical portions is 


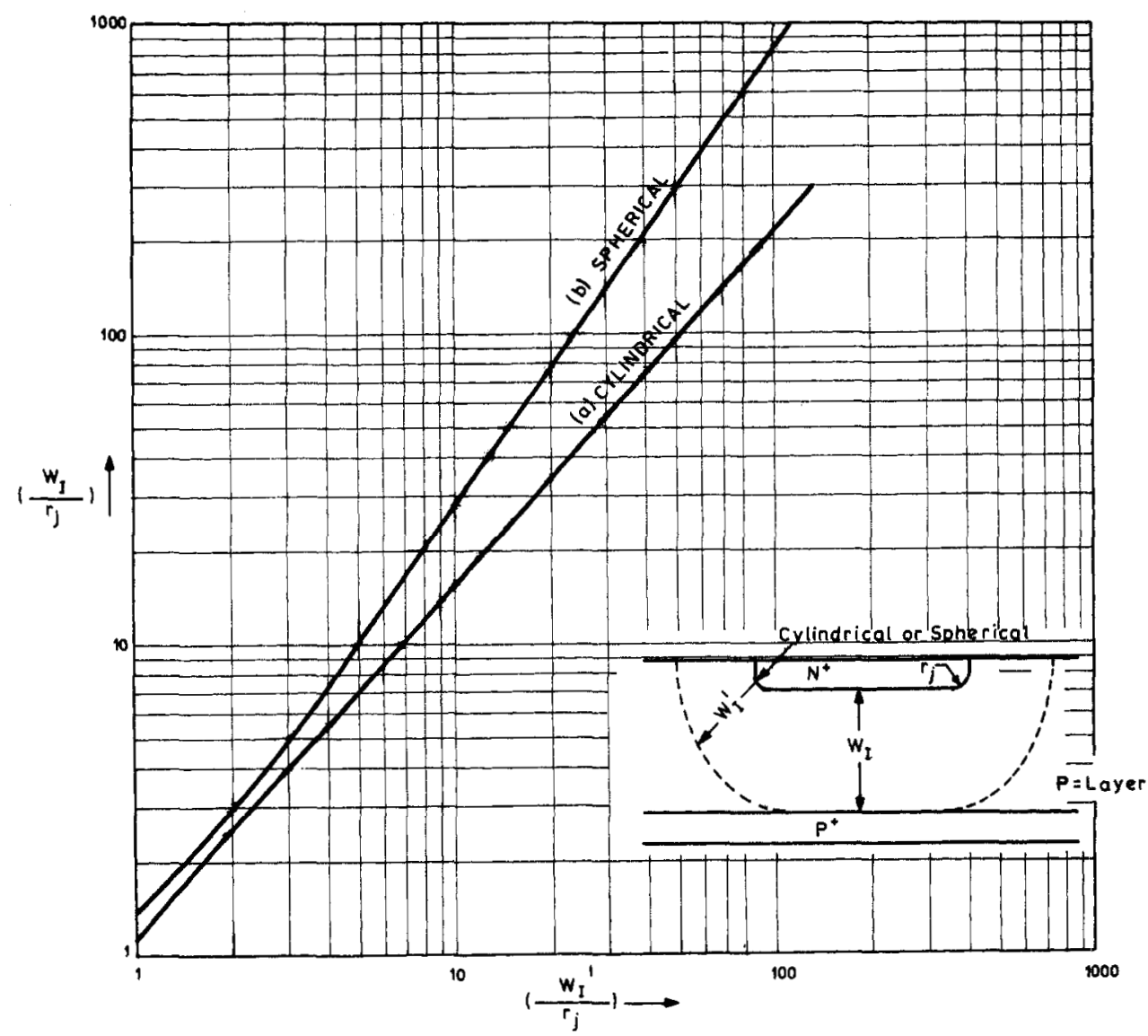

Fig. 3. Plot of the ratio of the width $W_{I}^{\prime}$ of depletion layer at punchthrough voltage $V_{P}$, to the radius of curvature $r_{j}$ against the ratio of middle layer width $W_{I}$ to $r_{j}$.

$W_{I}^{\prime}$. Now the electric field $E_{1 \mathrm{CY}}(r)$ in the cylindrical portion of the diode is given by the Gauss' law as follows:

$$
E_{1 C Y}(r)=\frac{q N_{I}}{2 \epsilon_{r} \epsilon_{0} r}\left[\left(r_{j}+W_{I}^{\prime}\right)^{2}-r^{2}\right]
$$

and the voltage across the depletion layer $V_{P}$ is

$$
\begin{aligned}
V_{P}= & \int_{r_{j}}^{r_{j}+W_{I}^{\prime}} E_{1 C Y}(r) d r=\frac{q N_{I}}{2 \epsilon_{r} \epsilon_{0}}\left[\left(r_{j}+W_{I}^{\prime}\right)^{2}\right. \\
& \left.\cdot \ln \left(1+\frac{W_{I}^{\prime}}{r_{j}}\right)-\frac{1}{2}\left\{\left(r_{j}+W_{I}^{\prime}\right)^{2}-r_{j}^{2}\right\}\right] .
\end{aligned}
$$

Substituting for $V_{P}$ from (2) in (9), we get the relation between $W_{I}$ and $W_{I}^{\prime}$

$$
\begin{aligned}
\left(\frac{W_{I}}{r_{j}}\right)^{2}= & \left(1+\frac{W_{I}^{\prime}}{r_{j}}\right)^{2} \ln \left(1+\frac{W_{I}^{\prime}}{r_{j}}\right) \\
& -\frac{1}{2}\left[\left(1+\frac{W_{I}^{\prime}}{r_{j}}\right)^{2}-1\right] .
\end{aligned}
$$

The $W_{I} / r_{j}$ ratio is calculated as a function of $W_{I}^{\prime} / r_{j}$ ratio and plotted in Fig. 3 (curve $(a)$ ) for cylindrical curvature ease.

When the applied voltage $V$ exceeds $V_{P}$, the electric field at the $\mathrm{p}^{+}-\mathrm{p}$ boundary becomes nonzero and the depletion region widens in the curved portions. In order to simplify analytical calculations, we assume that the additional voltage $\left(V-V_{P}\right)$ drops completely across the depletion layer which existed at voltage $V_{P}$. While this is strictly true only for the structure where the $\mathrm{p}^{+}-\mathrm{p}$ boundary has the shape of the depletion-layer boundary corresponding to voltage $V=V_{P}$, as discussed in the Appendix, most of the voltage drops within the width $W_{I}^{\prime}$ in the curved portions of the junction for the structure shown in Fig. 1(a). Therefore, we can write

$$
V-V_{P}=\int_{r_{j}}^{r_{j}+W_{I}^{\prime}} E_{2 \mathrm{CY}}(r) d r .
$$

By applying Gauss' law

$$
E_{2 \mathrm{CY}}(r)=\frac{E_{2 \mathrm{CY}}\left(r_{j}\right) r_{j}}{r}
$$

Using (12) in (11)

$$
V-V_{P}=E_{2 \mathrm{CY}}(r) r \ln \left(1+\frac{W_{I}^{\prime}}{r_{j}}\right)
$$

and

$$
E_{2 \mathrm{CY}}(r)=\frac{V-V_{P}}{\ln \left(1+W_{I}^{\prime} / r_{j}\right)} \frac{1}{r} .
$$

The total electric field $E_{\mathrm{CY}}(r)$ at any $r$, is given by

$$
E_{\mathrm{CY}}(r)=E_{1 \mathrm{CY}}(r)+E_{2 \mathrm{CY}}(r)
$$

where $E_{1 \mathrm{CY}}(r)$ is due to $V_{P}$ alone as given by (8). Using (2), (8), and (14) in (15) 


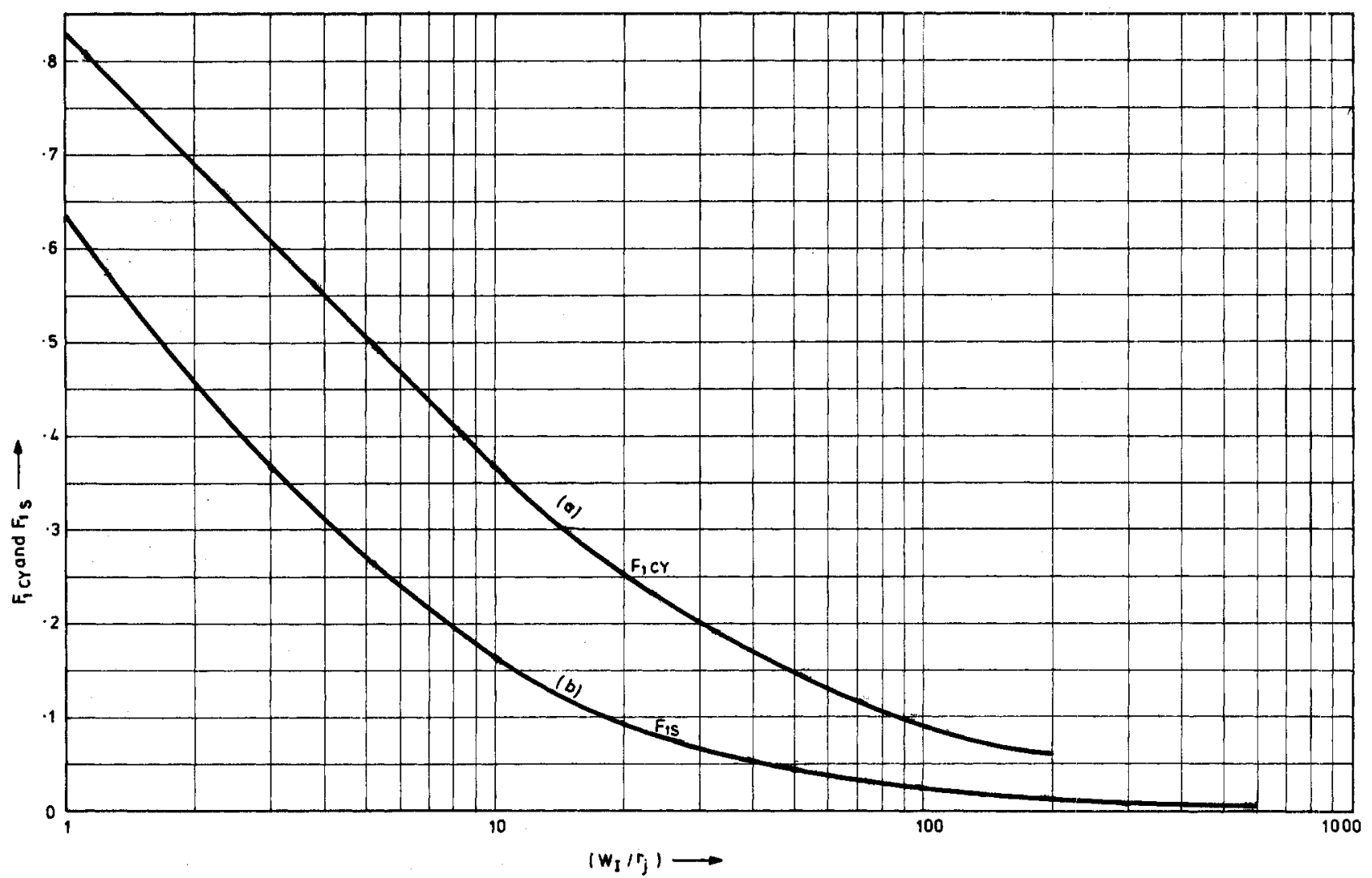
Fig. 4. Design curves for the determination of breakdown voltage of abrupt-cylindrical and spherical junction $\mathrm{p}^{+}-\mathrm{i}-\mathrm{n}^{+}$
diodes.

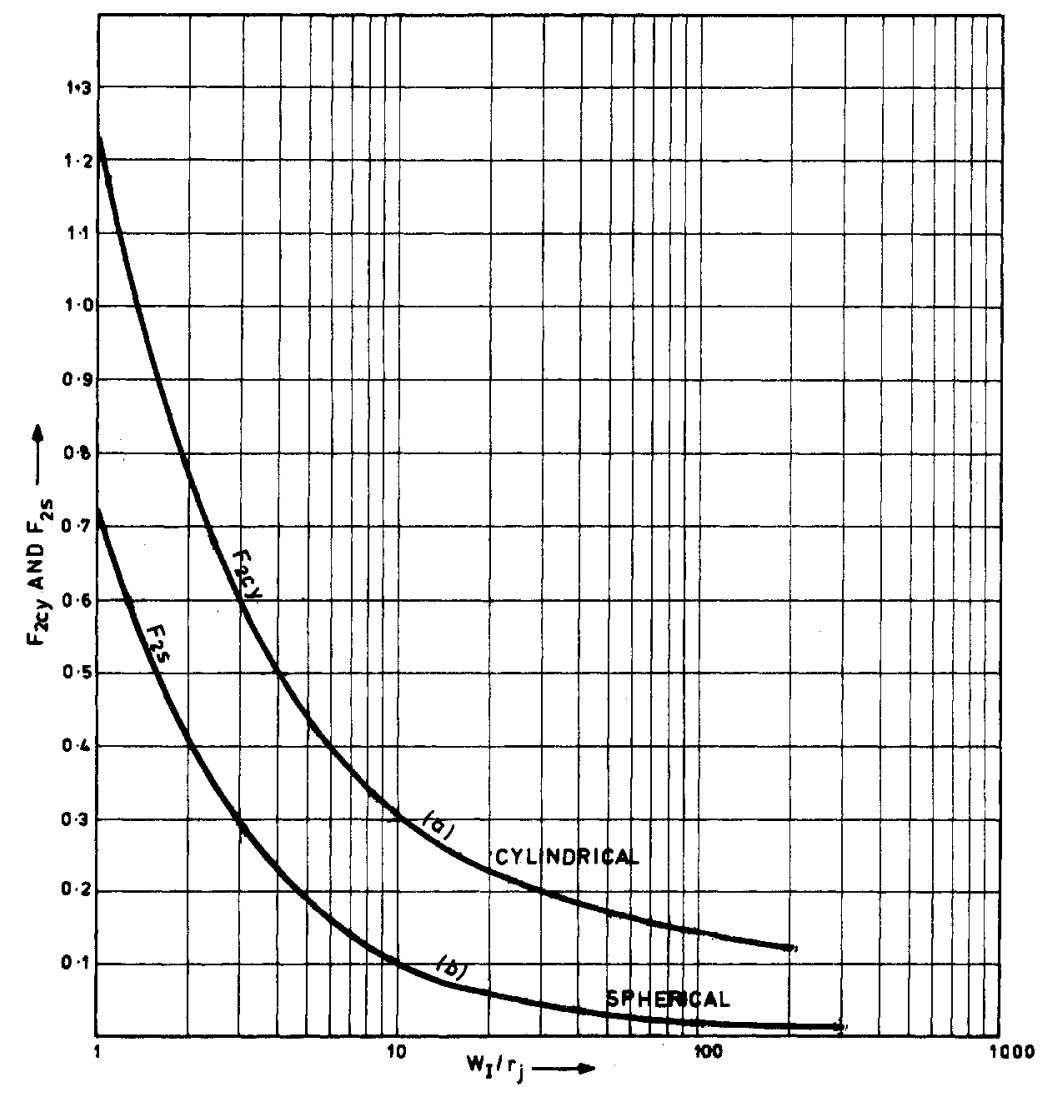

Fig. 5. Fraction of $V_{P}$ to be subtracted from $B V_{P P I} F_{1 \mathrm{CY}}$ and $B V_{P P I} F_{1 S}$ to obtain $B V_{C Y}$ and $B V_{S}$, respectively. 


$$
\begin{aligned}
E_{\mathrm{CY}}(r)= & {\left[\frac{V-V_{P}}{\ln \left(1+W_{I}^{\prime} / r_{j}\right)}+V_{P}\left(\frac{r_{j}}{W_{I}}\right)^{2}\left(1+\frac{W_{I}^{\prime}}{r_{j}}\right)^{2}\right] } \\
& \cdot \frac{1}{r}-\frac{V_{P}}{W_{I}^{2}} r .
\end{aligned}
$$

An examination of (16) shows that the contribution from the last term, $\left(V_{P} / W_{I}^{2}\right) r$, is small compared to other terms and can be ignored, so that (16) simplifies to the form

$$
E_{\mathrm{CY}}(r)=\frac{K_{\mathrm{CY}}}{r}
$$

where

$$
K_{\mathrm{CY}}=\frac{V-V_{P}}{\ln \left(1+W_{I}^{\prime} / r_{j}\right)}+V_{P}\left(\frac{r_{j}}{W_{I}}\right)^{2}\left(1+\frac{W_{I}^{\prime}}{r_{j}}\right)^{2} .
$$

Using (17) and (18) in the ionization integral

$$
\int_{r_{j}}^{r_{j}+W_{I}^{\prime}} A E_{\mathrm{CY}}^{7}(r) d r=1
$$

we obtain the breakdown voltage $B V_{\mathrm{CY}}$ as

$$
B V_{\mathrm{CY}}=F_{1 C Y} B V_{P P I}-F_{2 C Y} V_{P}
$$

where

$$
\begin{aligned}
F_{1 \mathrm{CY}} & =(6)^{1 / 7}\left(\frac{r_{j}}{W_{I}}\right)^{6 / 7} \frac{\left(1+W_{I}^{\prime} / r_{j}\right)^{6 / 7} \ln \left(1+W_{I}^{\prime} / r_{j}\right)}{\left[\left(1+W_{I}^{\prime} / r_{j}\right)^{6}-1\right]^{1 / 7}} \\
& \approx(6)^{1 / 7}\left(\frac{r_{j}}{W_{I}}\right)^{6 / 7} \ln \left(1+\frac{W_{I}^{\prime}}{r_{j}}\right)
\end{aligned}
$$

for $W_{I}^{\prime} / r_{i} \geqslant 1$, and

$$
F_{2 \mathrm{CY}}=\left[\left(\frac{r_{j}}{W_{I}}\right)^{2}\left(1+\frac{W_{I}^{\prime}}{r_{j}}\right)^{2} \ln \left(1+\frac{W_{I}^{\prime}}{r_{j}}\right)-1\right] \text {. }
$$

The factors $F_{1 C Y}$ and $F_{2 C Y}$ are plotted as curves $(a)$ in Figs. 4 and 5 , respectively, as a function of $W_{I} / r_{j}$. (The $W_{I}^{\prime} / r_{j}$ values required in (22) and (23) for a given $W_{I} / r_{j}$ are obtained from curve $(a)$ of Fig. 3.) These two plots together with the value of $B V_{P P I}$ obtained from (7) (plotted in Fig. 2) and of $V_{p}$ from (2) form the design curves for calculating the breakdownvoltage $B V_{\mathrm{CY}}$ of $\mathrm{p}^{+}-\mathrm{p}-\mathrm{n}^{+}$diodes having the structure shown in Fig. 1(a). For the case $V_{P}=0, F_{1 C Y} B V_{P P I}$ is the $B V_{\mathrm{CY}}$ of $\mathrm{p}^{+}-\mathrm{i}-\mathrm{n}^{+}$diodes.

\section{Punched-Through Diode with Plane Junction Terminated With SPHERICal Boundary}

Applying Gauss' law for the spherical case, the electric field $E_{1 S}(r)$ at any $r$ for a voltage $V_{P}$, is given by

$E_{1 S}(r)=\frac{2}{3} V_{P}\left(\frac{r_{j}}{W_{I}}\right)^{2} r_{j}\left[\left(1+\frac{W_{I}^{\prime}}{r_{j}}\right)^{3}-\left(\frac{r}{r_{j}}\right)^{3}\right] \frac{1}{r^{2}}$.

$W_{I}^{\prime}$ now stands for the depletion layer width at the spherical regions. Integrating $E_{1 S}(r)$ and equating it to $V_{P}$, as before, we get

$$
\left(\frac{W_{I}}{r_{j}}\right)^{2}=\frac{2}{3}\left(1+\frac{W_{I}^{\prime}}{r_{j}}\right)^{2}\left(\frac{W_{I}^{\prime}}{r_{j}}\right)-\frac{1}{3}\left[\left(1+\frac{W_{I}^{\prime}}{r_{j}}\right)^{2}-1\right] .
$$

TABLE I

Punched-Through Diodes (Having Cylindrical Junction) Fabricated USING $600-\Omega \cdot \mathrm{cm}$ p-TYPE SILICON WAFERS

\begin{tabular}{|c|c|c|c|c|}
\hline$W_{I}(\mu \mathrm{m})$ & $r_{j}(\mu \mathrm{m})$ & $W_{I} / r_{j}$ & \multicolumn{2}{|c|}{ Breakdown voltage (volts) } \\
\hline 155 & 10 & 15.5 & 700 & 680 \\
\hline 90 & 8 & 11.25 & 500 & 530 \\
\hline
\end{tabular}

Curve $(b)$ in Fig. 3 gives the plot of $W_{I} / r_{j}$ against $W_{I}^{\prime} / r_{j}$ for this spherical case.

Following the same procedure as in the cylindrical case, for voltages $V>V_{P},\left(V-V_{P}\right)$ produces an electric field $E_{2 S}(r)$ given by

$$
E_{2 S}(r)=\frac{\left(V-V_{P}\right) r_{j}\left(r_{j}+W_{I}^{\prime}\right)}{W_{I}^{\prime}} \frac{1}{r^{2}}
$$

and the total electric field $E_{S}(r)$ is the sum of $E_{1 S}(r)$ and $E_{2 S}(r)$ and approximates to

$$
E_{S}(r)=K_{S} / r^{2}
$$

where

$$
K_{S}=\frac{\left(V-V_{P}\right) r_{j}\left(r_{j}+W_{I}^{\prime}\right)}{W_{I}^{\prime}}+\frac{2}{3} \frac{V_{P}\left(r_{j}+W_{I}^{\prime}\right)^{3}}{W_{I}^{2}}
$$

Using (2) and (27) in the ionization integral, the breakdownvoltage $B V_{S}$ for the spherical case is given by

$$
B V_{S}=F_{1 S} B V_{P P I}-F_{2 S} V_{P}
$$

where

$$
\begin{aligned}
& F_{1 S}=(13)^{1 / 7} \frac{W_{I}^{\prime} / r_{j}}{\left[1+\left(W_{I}^{\prime} / r_{j}\right)\right]} \frac{1}{\left(W_{I} / r_{j}\right)^{6 / 7}} \\
& F_{2 S}=\left[\frac{2}{3} \frac{\left[1+\left(W_{I}^{\prime} / r_{j}\right)\right]^{2}\left(W_{I}^{\prime} / r_{j}\right)}{\left(W_{I} / r_{j}\right)^{2}}-1\right] .
\end{aligned}
$$

$F_{1 S}$ and $F_{2 S}$ are plotted in curves (b) of Figs. 4 and 5, respectively. (The $W_{I}^{\prime} / r_{j}$ ratio required in (30) and (31) for a given $W_{I} / r_{j}$ is obtained from curve (b) of Fig. 3 for the spherical case.) As in the cylindrical case, these two plots form the design curves for the calculation of breakdown voltages of punched-through diodes having spherical junction boundary. For the case $V_{P}=0, F_{1 S} B V_{P P I}$ is the $B V_{S}$ of $\mathrm{p}^{+}-\mathrm{i}-\mathrm{n}^{+}$diodes.

\section{Discussions AND CONCLUSIONS}

It is shown that the breakdown voltage $B V_{C Y}$ and $B V_{S}$ of punched-through diodes with cylindrical and spherical junction boundaries can be calculated with the help of two curves $F_{1 C Y}$ and $F_{2 \mathrm{CY}}$ (or $F_{1 S}$ and $F_{2 S}$ ) and a knowledge of $B V_{P P I}$ and $V_{P}$. Evaluation of $B V_{P P I}$ and $V_{P}$ are straightforward; e.g., $B V_{P P I}$ depends only on $W_{I}$ and is given in the form of a plot. $V_{P}$ has to be calculated using (2).

The design curves cover the range of values of $W_{I} / r_{j}$ to which most of the practical punched-through diodes fall. A couple of spot checks of breakdown-voltage measurements carried out on punched-through diodes fabricated using a $600 \Omega \cdot \mathrm{cm}$ re- 


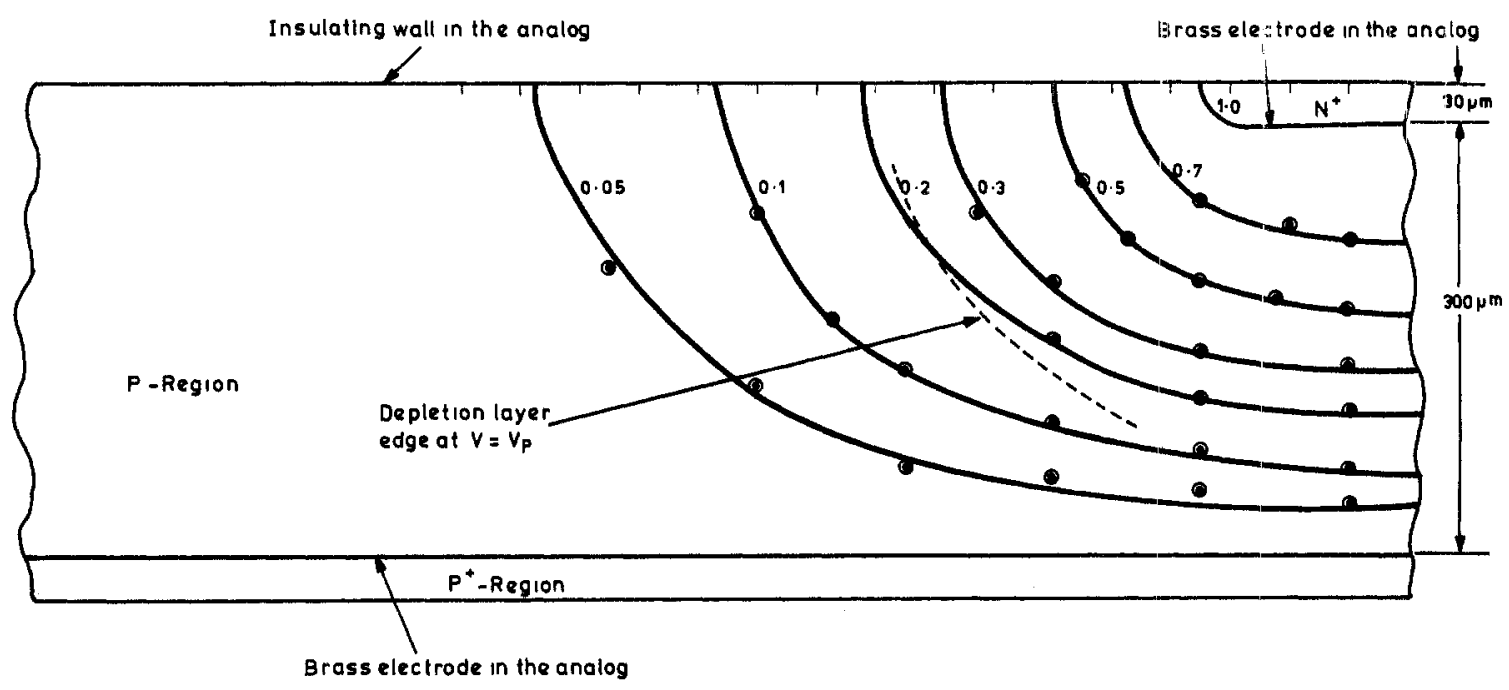

(a)

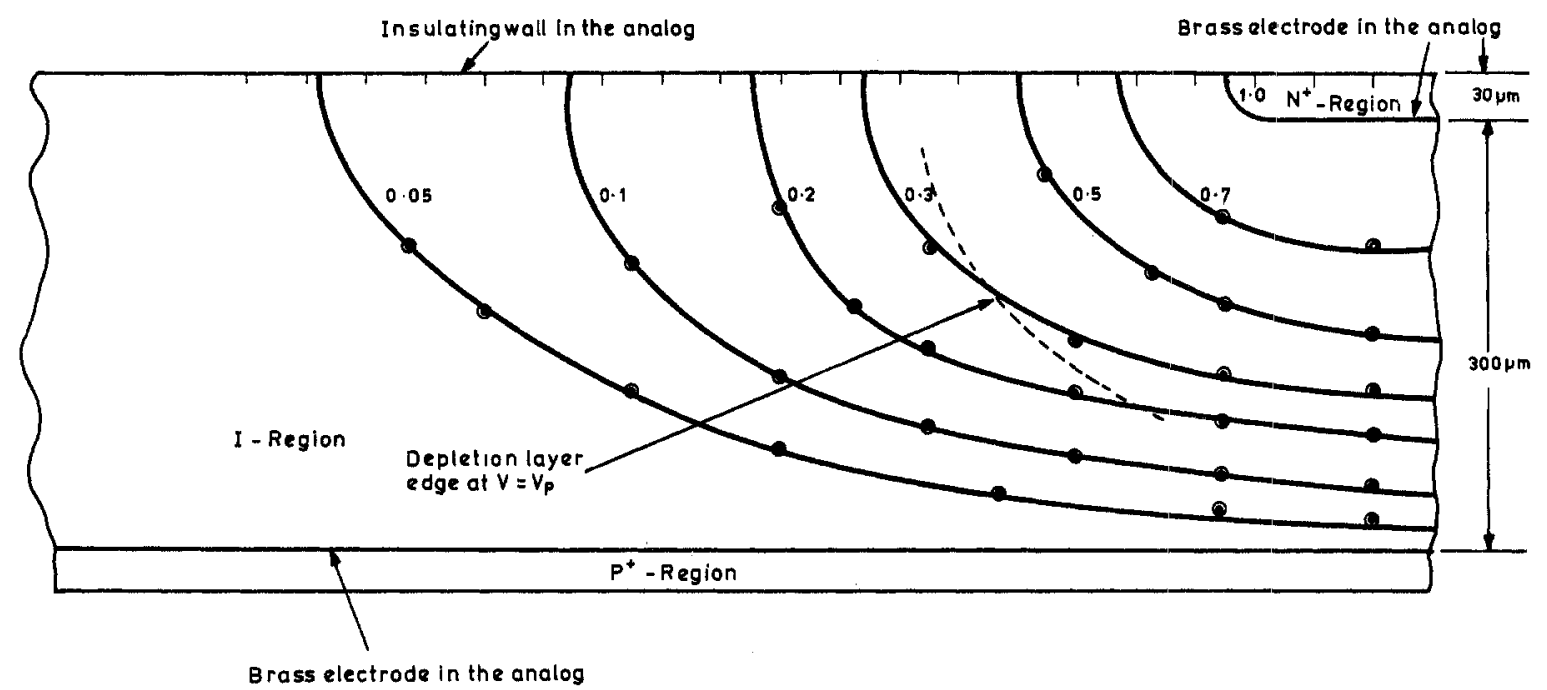

(b)

Fig. 6. (a) Potential plot in the $\mathrm{p}$ layer of $\mathrm{p}^{+}-\mathrm{p}-\mathrm{n}^{+}$diode biased beyond punchthrough voltage. (b) Potential plot in the $i$ layer of reverse-biased $\mathrm{p}^{+}-\mathrm{i}-\mathrm{n}^{+}$diode.

sistivity p-type silicon wafers are given in Table I. It may be noted that these values are in good agreement with the values estimated by the analytical solutions.

As discussed in the Appendix, the approximations involved are reasonable and enable the simplifications required for the analytical solutions.

\section{APPENDIX \\ Potential Distribution in the Middle Layer of Punched-Through Diodes}

In this section, the validity of approximations made in the previous sections regarding the voltage drop in the curved portion of the junctions is examined with the help of an electrolytic tank analog.

The potential $V$ in the $\mathrm{p}$ layer of a reverse-biased $\mathrm{p}^{+} \cdot \mathrm{p}-\mathrm{n}^{+}$ diode is given by

$$
\nabla^{2} V=\frac{q N_{a}}{\epsilon_{r} \epsilon_{0}}
$$

In order to obtain the potential distribution in the p-layer, we have made use of the electrolytic tank analog similar to the one which we had earlier used for transistors [13], [14]. Here, the $\mathrm{n}^{+}$and $\mathrm{p}^{+}$regions are represented by brass electrodes and thin copper pins are vertically mounted in the regions containing the electrolyte which represents the $p$ regions. Resistors are connected to these pins and the other end of the resistors are made common and kept at zero potential. A potential is applied to the electrode representing the $\mathrm{n}^{+}$boundary and the electrode representing the $\mathrm{p}^{+}$boundary is kept at zero potential. The potential distribution $\phi$ in the analog for this situation is given by [13], [14]

$$
\nabla^{2} \phi=\frac{\rho}{a t R} \phi
$$

where $\rho$ and $t$ are the resistivity and thickness, respectively, of the electrolyte, " $a$ " is the elemental area from which each pin drains current through the resistor $R$. The analog is made to a scale factor $K$ of 1000 . Equation (A1) in the diode gets modi- 
fied to an enlarged scale (for the same voltage) as

$$
\nabla^{2} V=\frac{q N_{a}}{\epsilon_{r} \epsilon_{0} K^{3}}
$$

Equations (A2) and (A3) are identical if $N_{a}$ is a function of position as potential $\phi$. Since the purpose of the present study is to obtain an idea of the potential distribution in the middle layer, we have taken this situation of varying $N_{a}$. Therefore

$$
\frac{q N_{a}}{\epsilon_{r} \epsilon_{0} K^{3}}=\frac{\rho \phi}{a t R}
$$

By choosing $a=1 \mathrm{~cm}^{2}, t=1 \mathrm{~cm}, R=1.35 \mathrm{M} \Omega$, and $\rho=2.5$ $\mathrm{k} \Omega \cdot \mathrm{cm}, \phi$ varying from $1 \mathrm{~V}$ to zero in the analog we have simulated the $\mathrm{p}^{+}-\mathrm{p}-\mathrm{n}^{+}$diode whose $N_{a}$ varies from $6 \times 10^{12} /$ $\mathrm{cm}^{3}$ to zero in the p region. Fig. 6 (a) gives the potential plot for this case for $r_{j}=30 \mu \mathrm{m}$ and $W_{I}=300 \mu \mathrm{m}$. The dotted lines show the boundary of radius of curvature $\left(r_{j}+W_{I}^{\prime}\right) ; W_{I}^{\prime}$ being $0.7 W_{I}$ as obtained from Fig. 3 curve $(a)$. It is clear that about 90 percent of the voltage drops within the width $W_{I}^{\prime}$ from the junction edge. Fig. $6(\mathrm{~b})$ gives the potential distribution for the case of $\mathrm{p}^{+}-\mathrm{i}-\mathrm{n}^{+}$diode (middle region purely intrinsic and hence the potential obeys Laplace equation. In the analog, the resistors $R$ are now disconnected to simulate this equation). It may be noted that even in this worse case, about 70 percent of the voltage drops within $W_{I}^{\prime}$ in the curved portions of the diode. Hence if the doping is greater than $6 \times 10^{12} / \mathrm{cm}^{3}$, the voltage drops within $W_{I}^{\prime}$ should be very close to the applied voltage. The validity of this analog study should hold good for other combinations of $r_{j}$ and $W_{I}$. Hence our assumption in the earlier sections that most of the voltage drops within $W_{I}^{\prime}$ is reasonably good for all doping concentrations of practical interest.

\section{REFERENCES}

[1] S. M. Sze and G. Gibbons, "Effect of junction curvature on breakdown voltage in semiconductors," Solid-State Electron., vol. 9, pp. 831-845, 1966.

[2] O. Leistiko and A. S. Grove, "Breakdown voltage of planar silicon junctions," Solid.State Electron., vol. 9, pp. 847 852, 1966.

[3] D. V. Speeney and G. P. Carey, "Experimental study of the effect of junction curvature on breakdown voltage in Si," SolidState Electron., vol. 10, pp. 177-182, 1967.

[4] R. M. Warner, "Avalanche breakdown in silicon diffused junctions," Solid-State Electron., vol. 15, pp. 1303 1318, 1972.

[5] D. A. Vincent, H. Rambeek, R. E. Thomas, R. M. Sirsi, and A. R Boothroyd, "Breakdown voltage of cylindrical Gaussian p-n junctions," Solid-State Electron., vol. 14, pp. 1193-1200, 1971.

[6] P. R. Wilson, "Avalanche breakdown voltage of diffused junctions in silicon," Solid-State Electron., vol. 16, pp. 991-998, 1973.

[7] D. P. Kennedy and R. R. O'Brien, "Avalanche breakdown calculations for a planar p-n junction," IBM J. Res. Devel., vol. 10, pp. 213-219, 1966.

[8] C. Bulucea, A. Rasu, and C. Postolache, "Surface breakdown in silicon planar junctions-A computer-aided experimental determination of the critical field," Solid-State Electron., vol. 17, pp. 881-888, 1974.

[9] V. A. K. Temple and N. S. Adler, "Calculation of the diffusion curvature related avalance breakdown in high voltage planar $\mathrm{p}-\mathrm{n}$ junctions," IEEE Trans. Electron Devices, vol. ED-22, pp. 910916, 1975.

[10] B. J. Baliga and S. K. Ghandhi, "Analytical solutions for the breakdown voltage of abrupt cylindrical and spherical junctions," Solid-State Electron., vol. 19, pp. 739-744, 1976.

[11] W. Fulop, "Calculation of avalanche breakdown voltages of silicon p-n junctions," Solid-State Electron., vol. 10, pp. 39-43, 1967.

[12] J. L. Moll, J. L. Su, and A. C. M. Wang, "Multiplication in collector junctions of silicon n-p-n transistors," IEEE Trans. Electron Devices, vol. ED-17, pp. 420-423, 1970.

[13] K. N. Bhat and M. K. Achuthan, "Current gain enhancement in lateral pnp transistors by an optimized gap in the $\mathrm{n}^{+}$buried layer," IEEE Trans. Electron Devices, vol. ED-24, pp. 205-214, 1977.

[14] $\frac{1}{-}$, "Transverse diffusion in planar transistors," IEEE Trans. Electron Devices, vol. ED-25, pp. 57-60, 1978. 